\title{
Hypertext as Method
}

\author{
Claus Atzenbeck* \\ Hof University \\ Institute of Information Systems \\ Hof, Germany \\ claus.atzenbeck@iisys.de
}

\author{
Peter J. Nürnberg \\ Neosententia, LLC \\ Austin, TX, USA \\ peter.j.nuernberg@gmail.com
}

\begin{abstract}
Historically, there has been a tendency to consider hypertext as a type of system, perhaps characterized by provision of links or other structure to users. In this paper, we consider hypertext as a method of inquiry, a way of viewing arbitrary systems. In this view, what are traditionally called "navigational hypertext systems" might be considered as information retrieval systems, "spatial hypertext systems" as brainstorming systems, etc., while their "hypertext" nature results from the way in which such systems are conceived, developed, and/or presented. The benefit of such a shift is the ability to apply this hypertextual method of inquiry to systems not normally considered part of the hypertext community. In this paper, we specifically apply this view to artificial intelligence, and examine how this application can be productive.
\end{abstract}

\section{CCS CONCEPTS}

- Human-centered computing $\rightarrow$ Hypertext / hypermedia; Graphical user interfaces; User centered design; - Software and its engineering $\rightarrow$ Software infrastructure.

\section{KEYWORDS}

hypertext; AI; augmentation; intellect; intelligence; man-machine; structures; context; infrastructure; research communities; hypertext history

\section{ACM Reference Format:}

Claus Atzenbeck and Peter J. Nürnberg. 2019. Hypertext as Method. In 30th ACM Conference on Hypertext and Social Media (HT '19), September 17-20, 2019, Hof, Germany. ACM, New York, NY, USA, 10 pages. https: //doi.org/10.1145/3342220.3343669

\section{INTRODUCTION}

There have been numerous definitions given for the term "hypertext" over the past several decades, including [8, 13, 22, 32, 37, 53]. We are interested here in a related but different topic: what are the defining characteristics of the hypertext community? The community has often been characterized as one that includes (and sometime integrates) a wide range of subjects, from human computer interaction [cf. 31, 36] to core technology [cf. 3, 48, 50],

\footnotetext{
${ }^{*}$ Corresponding author
}

Permission to make digital or hard copies of part or all of this work for personal or classroom use is granted without fee provided that copies are not made or distributed for profit or commercial advantage and that copies bear this notice and the full citation on the first page. Copyrights for third-party components of this work must be honored.

For all other uses, contact the owner/author(s).

HT '19, September 17-20, 2019, Hof, Germany

(C) 2019 Copyright held by the owner/author(s)

ACM ISBN 978-1-4503-6885-8/19/09.

https://doi.org/10.1145/3342220.3343669 from literary critical theory [cf. 1, 9, 27, 28, 52] to social media [cf. 39, 44, 45, 51, 54] and more. In what sense does this form a coherent whole? What ties this diverse set of fields together? How much have these common threads changed over time?

Though it may be impossible to answer these questions definitively, we feel there are commonalities that help define hypertext as a method of inquiry, a lens through which other topics are investigated. In this paper, we consider our method as built of two parts: the aim or goal of the work being considered; and, a set of perspectives used to describe or pattern thinking about the work. We do not claim that we have been exhaustive - there are certainly additional ways to characterize hypertext as method - but we show how our initial definition can be productive when analyzing a field not normally considered part of the orbit of hypertext: artificial intelligence.

The paper proceeds as follows. Section 2 addresses the first part of our method, augmentation, which has served as the aim or telos of much of the work done in the hypertext community over the past decades. Section 3 addresses the second part of our method, three perspectives or eide of structure that characterize the discussions in a wide range of hypertext literature. Section 4 demonstrates the utility of our method by applying it to artificial intelligence work. Section 5 reviews a system implementation driven by the philosophy and perspectives we describe. Finally, Section 6 provides directions for future work and our conclusions.

\section{AUGMENTATION}

Historically, much of hypertext literature described work that (implicitly or explicitly) was aimed at "augmenting the human intellect" [4, 13, 15, 49]. To continue with Engelbart: "By 'augmenting human intellect' we mean increasing the capability of a man to approach a complex problem situation, to gain comprehension to suit his particular needs, and to derive solutions to problems." [20]

This focus on the human intellect requires us to consider what parts of that intellect we are interested in augmenting. Much early hypertext literature often started with the notion that human thinking and reasoning is ultimately associative; there is no forced conformity either to strict structures such as hierarchies or to linear thinking. We all experience thoughts that suddenly appear in a discussion. Ideas pop up, ignoring structural boundaries in a nonlinear, non-hierarchical fashion.

An oft-cited example of this associativity is "intertextuality" [17]. Consider the Gospel of John, which refers in the scene of Jesus' death:

"After this, Jesus, knowing that all was now finished, said (to fulfill the Scripture), 'I thirst.' ” [16, John 19:28]

This refers implicitly to the Book of Psalms: 
"my strength is dried up like a potsherd, and my tongue sticks to my jaws; you lay me in the dust of death.” [16, Ps 22:15]

The reader of John's gospel needs to understand to what the insertion "to fulfill the Scripture" refers to. It is a reference without an explicit, unique pointer to the destination. Due to their nature, associations in natural language texts are mostly implicit or subtle. They require an understanding of their context and relations to other texts and may lead to ambiguities or alternate interpretations, which, in turn, makes it difficult for humans to recognize or identify relations. For machines, it still is impossible to grasp all notions, variants, implications, or connections of natural language texts. This is an important aspect that we will reconsider later.

Contrast this implicit, ambiguous structure with more rigidly defined (and more constrained) structure types such as lists, tables, or hierarchies, which are ubiquitous on computers; for example, file systems that contain folders and sub-folders, or contentmanagement systems (CMS) that impose hierarchical structures. XML and its widespread variants are also hierarchical.

Such structures were designed to reflect what people knew from file cabinets or physical spreadsheets. Adding associations freely and arbitrarily on paper used to be too inconvenient in order to become practicable in mainstream knowledge work. As Ted Nelson expressed:

"Hierarchy is not in the nature of the computer. It is in the nature of the people who set computers up. If you say, 'everything is hierarchical,' as many computer people do, that is not science, it's a metaphysical position. It can't be proven true or false, it can only be proven inconvenient." [35]

Before computers, as we know them today, became widespread, Vannevar Bush [10] realized that knowledge workers needed to handle increasingly huge amounts of information. He designed Memex, a tool for humans to store trails between documents persistently. In other words, with Memex, associations that knowledge workers encounter or create during their work with documents were persistently stored. Thus, they could be recalled any time later or given to colleagues for them to traverse the same paths.

As computers became more widespread, scientists, philosophers, and engineers such as Ted Nelson and Douglas Engelbart developed machines that were able to associate various information with links [cf. 21, 34]. Thus, it became possible to overcome some limitations of paper with respect to interlinking [32]. People were capable of expressing and storing thoughts (associations) explicitly by using machines, going beyond the previously implicitly expressed references that were interlaced in the text itself (intertextuality). With hypertext links, there is less overhead for people to express or communicate such associations.

Ted Nelson coined the term hypertext in 1965 to mean "a body of written or pictorial material interconnected in such a complex way that it could not conveniently be presented or represented on paper" [32]. Later he states in his book Computer Lib/Dream Machines "Everything is deeply intertwingled." [33] and refers to the fact that "all subjects and issues are intertwined and intermingled" [35]. Tim Berners-Lee argues along the same lines in his book Weaving the Web:
"In an extreme view, the world can be seen as only connections, nothing else. We think of a dictionary as the repository of meaning, but it defines words only in terms of other words. I liked the idea that a piece of information is really defined only by what it's related to, and how it's related. There really is little else to meaning. The structure is everything." [6]

As such, it is impossible to represent arbitrary associative information in simple structures, such as lists or hierarchies, without losing information; they are too simplistic to represent all of the nuances of human knowledge.

At his point we can summarize that hypertext is about associating information explicitly. This relates strongly to the human capability of thinking and the acknowledgement of the fact that structure can be found everywhere.

Beside the typical node-link structures, which became widely known by the Web, hypertext researchers considered other types of structure for freely associating information, escaping the tyranny of lists, hierarchies, or tables. Examples include spatial hypertext [29], taxonomic hypertext [40], or argumentation supporting structures [14].

With those structure types, associations are not expressed by links, but by other means. For example, spatial hypertext uses visual cues such as spatial distance or arrangements for expressing associations. This is not what many would expect from a computer, as this results in fuzzy connections, ambiguities, or different interpretations. Such implicit structures are more difficult to handle by machines [46], which can result in people not expecting to work with such structures on computers.

Machines may support human intellectual work. However, the use of links or generic graphs does not by itself make an application a hypertext system if it does not incorporate human intelligence or knowledge representation. For example, we would not consider a graph database a hypertext system only due to its feature of representing edges. Conklin points this out in his 1987 paper, which clearly refers to human users:

"It is tempting to describe the essence of hypertext as its ability to perform high-speed, branching transactions on textual chunks. But this is a little like describing the essence of a great meal by listing its ingredients. Perhaps a better description would focus on hypertext as a computer-based medium for thinking and communication." [13]

In recent decades, with increasing capabilities of information technologies, hypertext research has shifted toward a stronger focus on technologies and techniques. Some examples can be found in the Web (which provides unidirectional, embedded links), the semantic Web (which adds semantics on top of data and aims for semantic-aware machines), or social network and Twitter analytics (which target issues of data mining and machine learning). Those examples are supportive to human tasks; however, they do not strongly augment human capabilities. Along these lines, human capabilities have received less emphasis in more recent hypertext research. This was also mentioned by Engelbart in an interview with Belinda Barnet in 1999 [5]: 
Barnet: "Do you think people understand your 'framework' more now than they used to?"

Engelbart: "Not particularly, no. - The paradigms seem to be 'oh, we're going to automate things we do now, automate the way we do business' etc., and the idea of really augmenting, people are beginning to register, but it's in a limited conceptual frame."

In her HUMAN'18 keynote, Barnet further argues that "the design of deep data collection platforms like Facebook and the algorithms that make inferences based on that data for advertisers ('machine learning')" [4] even violate Engelbart's framework, as they are "skewed against human being" and, thus, are "disempowering".

It has become widely accepted that technologies that relate to the Web platform belong to the field of hypertext. It is no surprise that projects related to Twitter, which itself runs on the Web platform, are easily recognized as hypertext projects, even if they lack support of human thinking.

We have argued that hypertext bears the connection to augmenting human intellect, as described by the hypertext pioneers Bush, Engelbart, and Nelson. Only considering the link is not valid, as structure is everywhere. Would one argue chemistry is hypertext, because molecules show specific structures? Or would one argue that computer vision is hypertext, because some AI components identify certain structures on images or videos?

This way of thinking would create an inflation of the term "hypertext", which would make it meaningless. On the contrary, the focus on augmenting human intellect is more useful. It is backed up by almost 75 years of hypertext history and, thus, a well established point of view. Furthermore, it lets us distinguish hypertext from any other field of research dealing with structure outside a human scope. At the same time, hypertext makes use of such related technologies, including AI, text/data mining, various database types, or network technologies.

Thus, we conclude that hypertext mainly refers to its capability to support human intellectual capabilities and decision making processes rather than a specific technology or related principles. This said, there are two important aspects:

(1) Hypertext still needs massive support from technology-driven communities. For example, data analytics may be used in the context of augmentation.

(2) Hypertext is interdisciplinary by nature. This means that there are intersecting areas with many other fields of research or application.

\section{THREE PERSPECTIVES}

\subsection{Background}

In the previous section we argued that the aim of much hypertext work is augmentation. Historically, hypertext considers structure as a "tool" for augmenting, extending, and communicating human thinking. This is analogous to, for example, a hammer, which extends a human's arm to become harder, stronger, and heavier in order to increase its potential force.

There are a great number of forms of structure discussed in the hypertext literature, as mentioned and referred to in the previous section, including node-link, spatial, taxonomic, or argumentation supporting structures. The question arises about the characteristics of such structures.

In this section, we consider three perspectives that have been repeatedly expressed in the hypertext literature. We examine how they have been used to analyze a wide variety of work. We consider these characteristics of structure independent of its form.

Also, it is of course true that these perspectives are not unique to hypertext as a field. We view this as a matter of degree - these perspectives often co-occur and are well-documented in the hypertext literature; other fields may adopt features of some of these perspectives, but the primacy the hypertext literature places upon them is distinguishing.

\subsection{First Class Structure}

In a great variety of hypertext literature, structure is presented as a "first class" concept. This can take the form of "primary element of analysis" [30] to "explicit system object" [47]. Structure even plays a central element in several definitions of the term hypertext, such as [32].

The reification of structure allows us to consider possibilities not available to us in scenarios in which structure is merely implicit. In hypertext systems literature, first class structure is said to ease features such as structure sharing [41] and structure versioning [55]. In hypertext literature analysis, the structures of criticism are made explicit [30].

Consider systems such as Construct [57], the entire architecture of which is centered around middleware services that provide different types of structure (e.g., navigational node-link, spatial, etc.). As first-class system objects, the structure abstractions served by this middleware can in turn also be structured. Consider further XLink, the linking elements of which are described as "explicit relationship[s] between resources or portions of resources" [18]. This allows the fulfillment of one of the design goals of the standard, namely, the ability to "[a]ssociate metadata with a link".

Of course, many fields discuss the structure of data. However, outside of hypertext, structure is often seen as implicit in the data in question. (This is even true in some system generally considered as examples of hypertext systems, such as the WWW, in which links are often implicitly defined only in terms of explicit start and end points.) The hypertext view of first class structure allows us potentially to present enhanced features and characteristics of the structure that would otherwise not be available.

\subsection{Context Dependent Structure}

Another common theme in hypertext literature is the notion that structure of data is dependent on the user/reader, the time, the task, or a particular point of view. That is, data is not inherently structured, but rather the structure is appropriate both to the data and the context in which it is being viewed.

The nature of the context considered varies significantly throughout the hypertext literature. In the WWW, explicit structure can be realized through standards such as XLink [18]. Spatial hypertext systems often consider multiple parses of a particular space [cf. 46]. In hypertext literary work, there are discussions of traversaldependent links [cf. 7, 8, 11], in which some links are available based on the traversal history of the reader. 
Work in other fields addresses of context-dependent structure. For example, aspect-oriented programming considers ways in which code structured as "aspects" can be woven into codebases structured in other ways, such as traditionally object-oriented code [26]. Nonetheless, the hypertext literature stresses the non-primacy of any particular structure much more explicitly than in other fields.

\subsection{Open Ended Structure}

The final common thread running through the hypertext literature that we consider here is that structure is often (though not always) open ended. That is, it can be difficult or impossible to circumscribe the totality of structure that applies to a particular piece of data.

This "lack of closure" as a characteristic of hypertext literary work has been often cited [e.g. 19, 25]. It also brings to mind Nelson's notion that "Everything is Deeply Intertwingled" [33]. Some hypertext systems emphasize dynamically computed structure [57], which often implies open ended sets of structure, while models such as XLink [18] enable the addition of structure over content through the specification of external linkbases. Open structure implies change over time to the structure available over given content, a notion which has been discussed and even leveraged in hypertext literary analysis [30]. This openness stands in direct opposition to the more familiar notion of closed structure as evidenced in systems that use embedded structure (e.g., XML) or even traditional SQL DBMS.

In some ways, the acknowledgement of open ended structure follows from context dependent structure. If we allow that we cannot know all the contexts in which content may be used, we also cannot circumscribe all of the structure applicable to that content.

\section{HYPERTEXT AND AI}

We started our discussion with the focus in hypertext on augmenting human capabilities, specifically, human intellect. We have argued that hypertext should neither be seen as a specific technology (e.g., Web technologies) nor as a specific structure type (e.g., node-link). Either of those would limit the notion of hypertext and exclude certain research. The three perspectives above are ways of thinking about structure from a hypertext perspective, and are also independent of technologies or structure type.

Following this path, the following question arises: What role does computation - in particular artificial intelligence - take in the context of hypertext? How does AI match the presented idea of augmentation? What are the connections to the discussed perspectives on structure? How would hypertext and AI go together?

There is no doubt that computation has a history in hypertext. Even though in its very beginning, hypertext was often recognized as another way to access or navigate data, computation in hypertext systems has been discussed quite some time ago. A famous example is Frank Halasz' 1987 paper "Reflections on NoteCards" [24] in which he describes virtual, dynamic structures and computation over hypermedia networks.

Some of the issues raised in this context are directly related to the three perspectives of hypertext, as discussed in Sect. 3. For example, Halasz argues that "links are not first class objects in NoteCards, it is not possible to trigger computations during the access or modification of links" [24]”. Furthermore, Halasz' argument for virtual, dynamic structures that "would be defined intensionally, i.e., by specifying a description of their components" [24] is related to our discussion on context and open ended structures.

$\mathrm{AI}$ is a huge topic in today's scientific and societal discussions and developments. Examples include many current or future products or services such as autonomously driving vehicles, intelligent machines in the "industry 4.0" context, or systems predicting traffic jams or political decision making. They also include virtual assistants (e.g., Apple's Siri, Amazon's Alexa, or Microsoft's Cortana) that are already widespread today and accessible through smartphones, smart speakers, or modern OS. They are capable of "foreseeing" users' behavior (e.g., proposing the opening of an audio book player when a person leaves home) based on some regularity the system computed from the user's previous behavior.

Previous years have seen an increasing number of papers about data mining or machine leaning presented at hypertext related conferences. For example, the 2018 ACM Hypertext conference* had dedicated sessions on computational social science; machine learning and recommender systems; privacy, bots and automatic methods; and, news and community detection. Those topics made up the largest part of the conference. This example shows in an impressive way the importance of data mining or machine intelligence to the community, which goes hand in hand with the society's acceptance of such methods in our daily life.

In the examples mentioned above, AI mostly helps in automating tasks or workflows. The machine replaces the human in a sense, taking his/her role in completing the task. For example, a selfdriving car automates what the person would have to do manually; namely, operating the vehicle such that it carries its occupants to a specific destination.

While manually driving can be seen as a complex example of augmenting human capabilities (in particular capabilities related to or required for walking), autonomous driving is rather automation. This argument applies for many AI based products or services today. For example, recommender systems on sales platforms suggest products that the user may also want to buy. The user, however, does not have the possibility to augment his/her associations. The system limits the user to only perceive the provided information and possibly follow the suggestion. Thus, the machine automates the search for related information, but ignores the user's thoughts.

In the area of maintenance, the computation over huge amounts of data collected by industry robots or machines lets software decide what steps have to be taken in order to avoid upcoming failures. In personal discussions, CEOs or CTOs of small or medium industry enterprises have argued that with increasingly more "intelligent" machines that are capable of predictive maintenance, the need for highly educated staff is reduced and hiring less educated people who execute what the machine tells them becomes sufficient.

Hypertext contributes a different point of view. As argued in the previous sections, it supports augmentation rather than automation. Nevertheless, AI, data mining, or machine learning are important. As we will show by example in the next section, it is a matter of how a system can provide better decision making support by

*See https://dl.acm.org/citation.cfm?id=3209542 


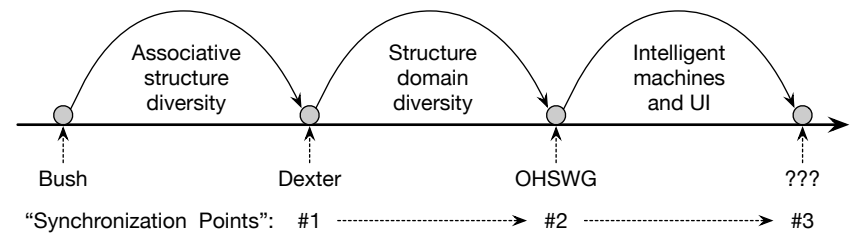

Figure 1: Synchronization points in hypertext research, taken from [3], extending [56]

considering human and machine as two cooperating sides. As such, humans are acknowledged to be the leading parties rather than the recipient of commands. Their capability of using associations, emotions, experience, or tacit knowledge is complementary to the machine's capability of quickly processing huge amounts of data.

The machine's recommendations to a given problem would be considered as suggestions to the user that can be further put into relationship or context, reflecting the human's thinking. The automation part is reduced to background computation assisting the user's search for information. The yet often lacking feature of systems is a way to let users associate information (including that proposed by machines).

Thus, hypertext can contribute a point of view to a variety of communities which is not yet seen much in today's political or societal discussions or in the products or services in our daily life. $\mathrm{AI}$ in the context of hypertext, as proposed in this section, becomes supportive in augmenting human intellect rather than automation. Its main purpose is to trigger human associations, i.e., to inform the user of potentially related issues of which the user may pick some that fit into his/her thinking.

An often overlooked beneficial side effect is that giving users the means to express their ideas or thinking may be taken as contributions to all. This is possible if the human is not only recipient of machine created recommendations, but can express human thinking, emotions, or intuitions easily. It would be complementary to the machine's knowledge discovery and could become part of the machine's overall knowledge from which recommendations are computed. Thus, hypertext is capable of capturing the intellectual or emotional intelligence of the masses and merge this with machine intelligence for better problem solving.

In an earlier paper [3] we extend the synchronization points in hypertext research suggested by [56] with intelligence machines and intelligent UI. Figure 1 represents an overview. It acknowledges automation as one of the main research directions in various fields and combines it with the original hypertext idea of augmentation. This enlightens a niche for hypertext which is unique: a method that may be applied to various fields, combined with a variety of other research directions of which $\mathrm{AI}$ takes one of the most important and prominent roles.

\section{APPLICATION DOMAINS}

\subsection{Background}

As mentioned in the previous sections, there are several structure types available that have been considered in hypertext research, including node-link and spatial hypertext. The core of hypertext

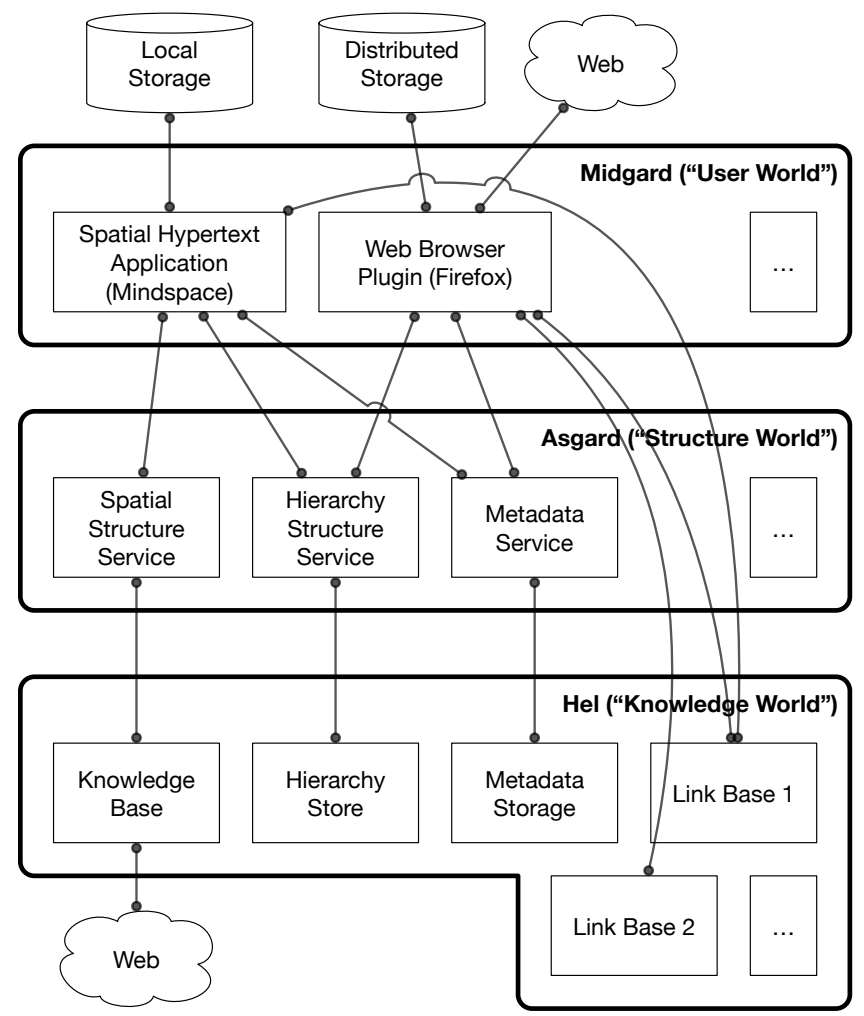

Figure 2: Mother's overall architecture with suggested connections between components (not all implemented)

is its capability to augment human thinking. Augmentation in this sense refers to the capability to associate information or ideas. We argue that this lifts up problem solving to a new level, as it allows human users to contribute their tacit knowledge, intuition, experiences, or ethical and moral thinking; and machines provide their capability of computing huge amounts of information and finding relevant information quickly.

In this section we discuss three projects in the context of hypermedia, two already finished ("HEIMDALL" and "DemoMedia"), the other one in its early phase ("IWInxt"). All three projects were executed at the Institute of Information Systems at Hof University. They are based on the component-based open hypermedia system (CB-OHS) Mother. Mother is an infrastructure supporting arbitrary structure services, including spatial hypertext, node-link structures, or metadata. The most distinguished feature in Mother is its spatial parsers that are capable of computing associations between nodes placed on a 2D canvas. This is based on their visual cues, spatial arrangements, or distance [46]. Figure 2 shows Mother's architecture. It consists of three layers named after places in Norse mythology:

Midgard This layer includes all software components that are closely related to the human user. Those could be spatial hypertext GUI applications, link plugins for Web browsers, or mobile applications that provide means for information classification.

Asgard This includes Mother's structure aware components, for example, the spatial structure service with various parsers 
that compute associations between nodes. It also may host, for example, hierarchy structure, metadata, or link services. However, as argued in [2], the latter is currently implemented as a minimal service that only consists of link bases. They belong to the Hel software layer.

Hel This is the machine's knowledge layer. It includes knowledge bases, which form an essential part in Mother's intelligence. They contain relevant information that is called by the system and presented as suggested information to the user. This layer includes linkbases, metadata, or other storage components.

Any other data facility, for example, data mining methods used to populate knowledge bases are outside Mother's scope. The same is true for connected cloud or distributed storage facilities that let users save or share their work with others. Mother's architecture is described in greater detail in previous publications [cf. 2, 3].

The machine's interpretation of the knowledge space is in a way similar to the user's understanding. This is the foundation for teaming up both sides for better problem solving. We consider this an augmentation of human intellectual capabilities, as Douglas Engelbart presented them in his augmentation framework [20]. In our projects, the "Man-Artifact Interface", as Engelbart calls it, is primarily provided by Mother's "intelligent" spatial hypertext and enhanced with the various other services that provide means for adding links or metadata to arbitrary objects.

In order to provide a better understanding of Mother in the context of hypertext domains, we will further discuss three projects in the following sections. We will emphasize the system's capability to provide machine intelligence and support human augmentation at the same time. As such, the human user is not simply degraded to a recipient in favor of some automation providing AI or respective platforms; rather, $\mathrm{AI}$ is used for augmenting human thinking.

\subsection{Example 1: Project "HEIMDALL"}

HEIMDALL is a sub-project of ODIN ("Open Data Innovation"), funded by the German Federal Ministry of Education and Research. It focuses on an intelligent user interface to be used by data analysts (mainly non-programmers) for processing open data. The project is explained in greater detail in previous publications [cf. 3, 42].

ODIN and HEIMDALL are mainly based on Web technologies. They focus on software components, called Cubbles, which exist in repositories from which data analysts can select them. Cubbles are used to model data flows or data processing in order to solve a given problem at hand.

In order to achieve this, Cubbles can be "piped" together. Figure 3 depicts the BDE ("Browser-based Development Environment") with two Cubbles on the right hand side. The arrow between them indicates that the component "pref-school" outputs data that becomes the input for "pref-student".

Using the standard BDE without HEIMDALL extensions, the user would normally search for Cubbles using the search field or browse the repository for appropriate components. This becomes difficult if the analyst does not know explicitly what he/she is looking for. On the other side, the machine does not know the problem the user is trying to solve and, thus, is unable to propose a solution.
HEIMDALL's intelligent UI closes this gap: Mother's layer $\mathrm{Hel}$ includes knowledge bases that contain information about how Cubbles are related to each other. The spatial structure service in Mother's software layer Asgard uses spatio-temporal parsers [46] that analyze relations between components that the user has dropped onto the BDE. From that, the system acquires related Cubbles from the knowledge base and presents them to the user. Figure 3 depicts those suggested Cubbles as a prioritized list on the left hand side within the BDE. These recommendations inform the user of potentially useful components without him/her explicitly searching for them.

From an augmentation point of view, as discussed in previous sections, the machine augments the user's capabilities of finding and associating information. The user is still in full control: he/she is the one who decides which Cubbles are added to the space. Furthermore, it is his/her choice to decide upon the spatial layout of the Cubbles, which expresses the associations between or combinations of Cubbles.

Mother's capabilities of "understanding" user created knowledge, expressed by Cubbles placed on a 2D space in a meaningful manner, is different from many AI applications. The most distinguishable feature is that users can express their associations to which the machine reacts, and vice versa. The user is not reduced to a "recipient of orders", but rather augmented with associations that exist outside of his/her mind.

\subsection{Example 2: Project "DemoMedia"}

DemoMedia is a research demonstrator based on our Mother infrastructure that brought the idea of intelligent spatial hypertext user interfaces to the domain of entertainment. This project was conducted in cooperation with Loewe Technologies GmbH, a German manufacturer of electronic consumer and communication products. DemoMedia targets the question of how the user experience for smart TV can be improved toward recommending information. Furthermore, it provides means to users for expressing his/her own associations related to movies.

In particular the latter aspect is underrepresented in today's broadcasters' media collections. Those are capable of suggesting movies, TV shows, or documentaries to users; however, they lack of options for organizing, structuring or associating them.

For example, one of DemoMedia's use cases that was developed mentions an elderly lady who frequently watches documentaries about flowers and gardening. In order to get most out of those, she takes notes on paper in order to organize her thoughts. This clearly shows a gap in the used media and knowledge transfer: the knowledge she builds up is not considered by the broadcasters' recommender systems. Furthermore, the lady must "transform" what she sees on the screen to what she writes or what she puts in relation.

In order to close this gap, we propose a spatial hypertext system that lets users organize content. Furthermore, the system places recommended movies or other related information onto the space. Figure 4 shows a screenshot of the DemoMedia client: Larger nodes were placed by the user and organized spatially. The parsers then calculate their relationships and retrieve relevant information from the knowledge bases which gets placed in the 2D space. The user 


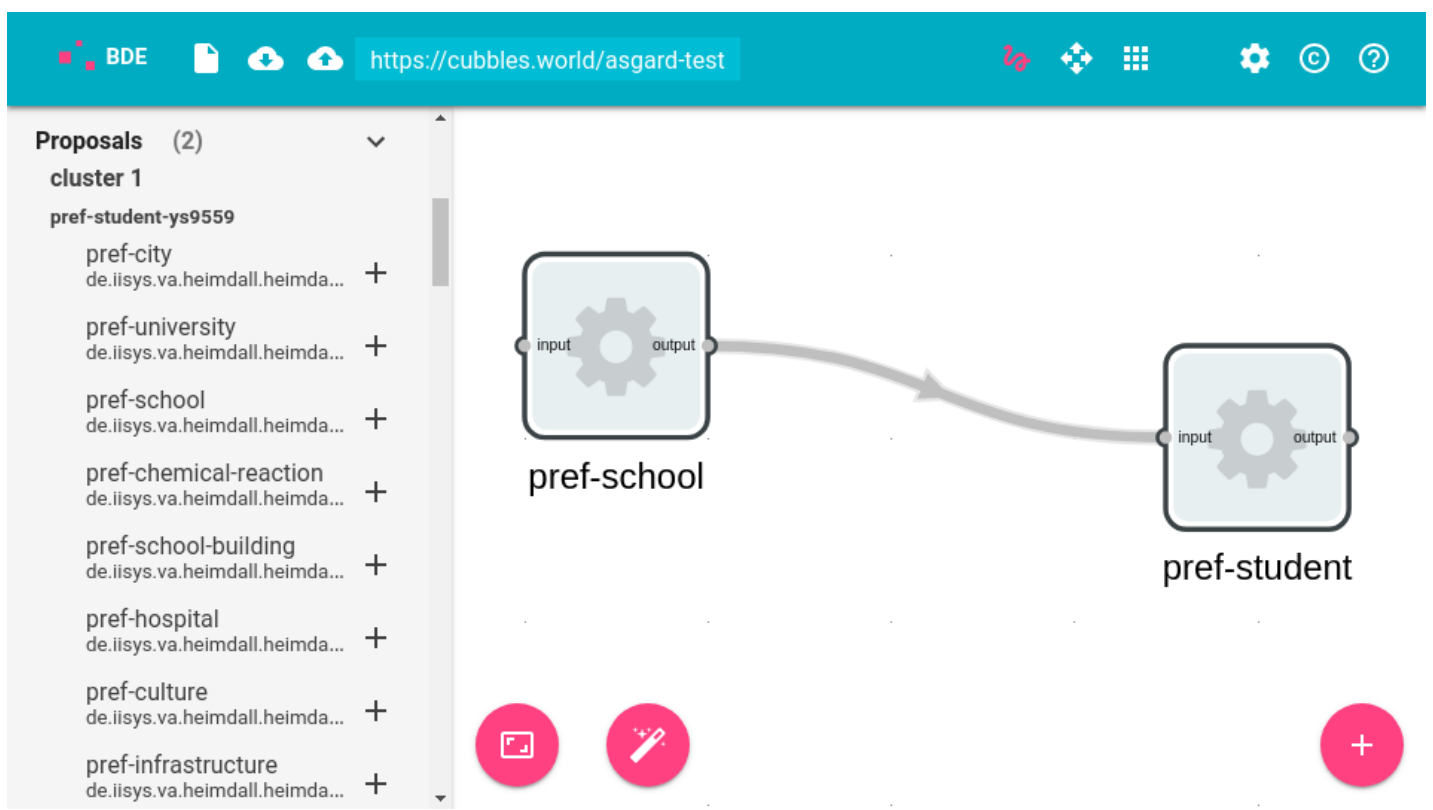

Figure 3: HEIMDALL's preliminary UI (taken from [42]): user added Cubbles (right) and prioritized list of suggested Cubbles (left)

may now chose to add some of the suggested information onto his/her space. Currently, there exist two knowledge bases (implemented in Neo4j) with movie metainformation taken from IMDB and Gracenote. Currently, 9,582 nodes and 34,204 edges have been selected to be used by the demonstrator.

The placement algorithm for suggested nodes is based on a spring metaphor such that any node "pulls" or "pushes" other nodes based on their relationship strengths. The underlying physics engine provides a natural look and feel of the objects during user interaction. The colors used in the figure indicate computed clusters. This is only used in the current client version for debugging purposes, as it allows the developers to "see" the system's parser results. (The visualization used in DemoMedia is discussed in greater detail in [43].)

This interface closes the media gap and supports a shared understanding of topics and interests for users and machine, as it provides means to a user to directly add information suggested by the system and, at the same time, lets the machine analyze the user's represented knowledge. It is another example of augmentation, as described by Douglas Engelbart.

\subsection{Example 3: Project "IWInxt"}

A third example is the recently started project IWInxt ("Next Generation Intelligent Maintenance System for the Industry 4.0"). It aims at supporting problem solving for maintenance staff.

Industrial machines are becoming increasingly intelligent. They produce masses of data which then are analyzed by computers in order to predict expected failures. This is called predictive maintenance; there is also corrective maintenance, which aims at solving problems that occurred unexpectedly [12].
Over time, maintenance workers build up experience which is hard to represent formally in its entirety. Writing down the ideas and steps someone took to solve a particular problem would entail tremendous cognitive overhead. Consequently, final reports often include a small portion of what actually lead to a problem's solution. Furthermore, if an experienced worker leaves a company, much of his/her tacit knowledge becomes unaccessible for the corporation.

We can solve some of those issues with an interface with which the maintenance staff can easily express individual thoughts within his/her problem solving strategy. In IWInxt, we propose a spatial hypertext client, as it permits structuring or restructuring of information or thoughts with little cognitive overhead. It is as easy as writing ideas on paper snippets and organizing them spatially on a desk. Furthermore, given our specialized parsers, the machine is able to capture individual steps over time, as well as any temporary or persistent relationship between information as expressed by the user.

With this, knowledge that the user produces or expresses during a decision making process can be persistently stored and communicated to other colleagues. At the same time, the system provides the user with additional information from the knowledge base, possibly enriched with information that comes from colleagues who worked on similar issues. Furthermore, the knowledge that has been expressed by staff becomes persistently available through the system. Thus, it stays available even after an employee has left the company.

IWInxt aims at extending human intellect, namely a user's capability of problem solving. It extends an individual's access to knowledge toward machine generated knowledge and includes indirectly also knowledge from colleagues using the same system. 


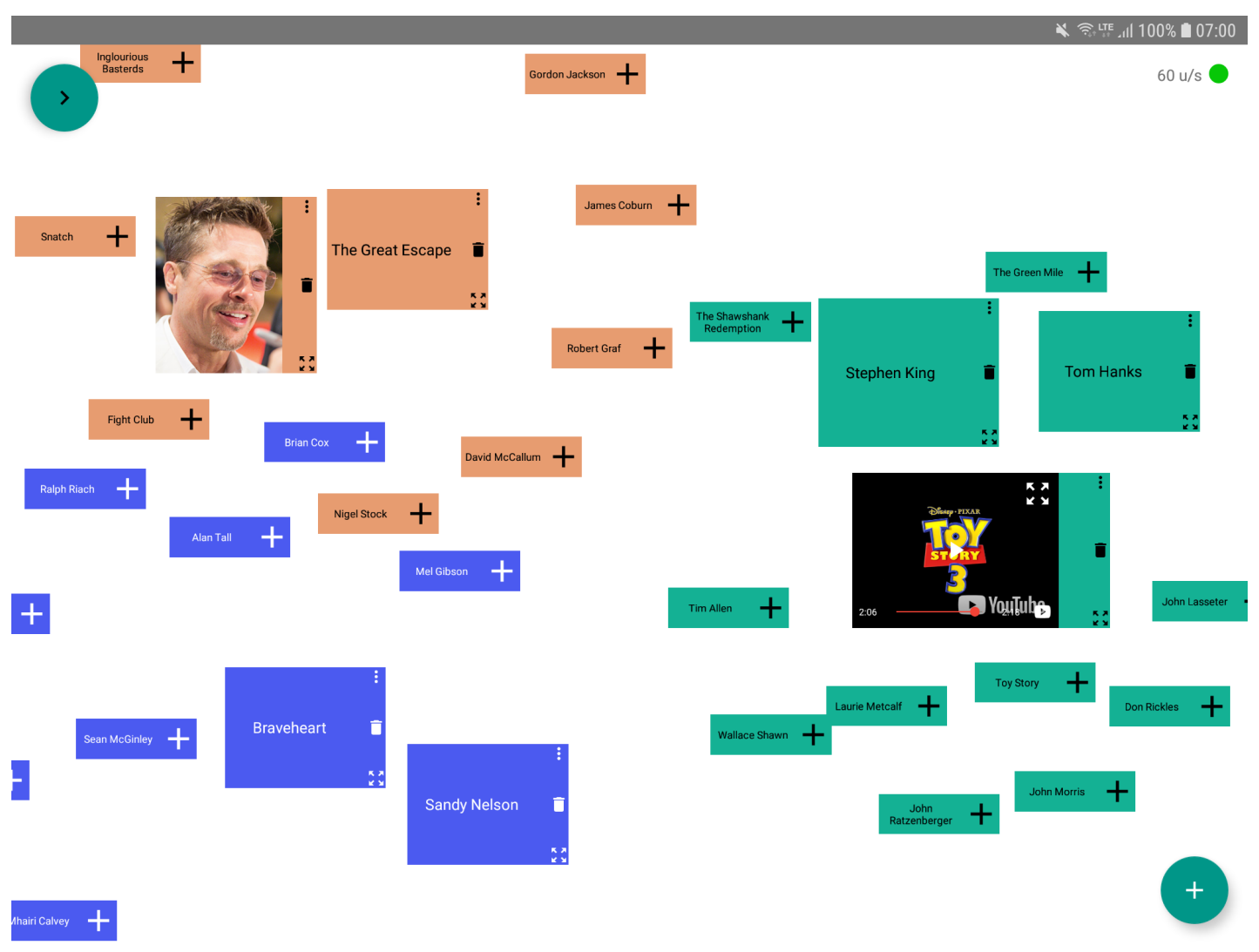

Figure 4: Screenshot of DemoMedia running on Android (photo of Brad Pitt by Dick Thomas Johnson, licensed under CC BY 2.0, extracted and cropped from https://flic.kr/p/21pNYLs)

\subsection{Reflections on the Given Examples}

As indicated in their respective sections above, all three projects aim at extending human capabilities. They close the gap between the user building up knowledge and the machine's capability of computing masses of data. Furthermore, they create a collaborative momentum, as the system is capable of capturing and relating knowledge expressed by multiple users. This also includes their tacit knowledge.

As argued in Sect. 3, hypertext provides means to capture and represent various structure types. The support and capability of representing associations is essential to augment human intellect. $\mathrm{AI}$ is currently a big topic that will become an increasing part of our daily lives. The examples above indicate that a close cooperation between human and machine improves problem solving. Each side should do what it can do best. That is, for humans, contributing their tacit knowledge and experiences; and, for machines, providing computation over huge amounts of data. The projects mentioned above aim at increasing the quality and effectiveness of problem solving by combining those distinct capabilities.

Even though the focus of the systems discussed above is their spatial structures, we have implemented various other structure types as well, including metadata and link support [2]. As a CB-OHS Mother is capable of handing arbitrary structure services. Structure is separated from data. For example, annotations or metadata is separated from nodes; Mother's linking model is based on Dexter [23] and handles connections (i.e., links) outside documents. Links are independent objects, which even can be annotated. Furthermore, links can be added to documents for which users do not have write access.

With respect to the spatial structure, Mother's specialized parsers create explicit knowledge graphs from implicit associations. This graph is maintained outside the nodes of a given spatial hypertext. This creation of "virtual structures" is a behavior that early hypertext systems were lacking of [24] and is still unsupported in most applications today.

Even though, Mother features first class structures, it does not support flexible structure-data boundaries as proposed, for example, with the EAD model [38].

Beside first class structure support, all specialized Mother instances support context dependent structures. For example, consider the automatically queried suggestion nodes that are presented to a user on the spatial hypertext canvas. First, the context of nodes is computed by the spatio-temporal parsers. Then, the structure is used to compile queries to the knowledge bases. The result is a context (among multiple possible) of the user's nodes, taken from the content of the knowledge bases. This makes both the user's and the machine's structures mutually dependent; one becomes the context of the other in each case. 
Mother's support for first class structures, (computed) context, and various structure types opens another level of freedom to users: Contexts can be changed easily, new structures computed, links or metadata added to any object that has an ID within or that is addressable outside the system, for example, Web pages by their URIs. Thus, Mother fosters open ended structures and multiple perspectives on data, intending to augment humans' capabilities in thinking, problem solving, communication, and cooperation.

\section{CONCLUSION AND FUTURE WORK}

We have discussed the idea that the notion of augmentation is central to hypertext undertakings. While we can of course talk of hypertext systems, it is at times productive to consider arbitrary systems from a hypertext point of view, starting with a notion of human augmentation, and concretely considering what benefits might obtain from considering first-class structure, context-dependent structure, and/or open ended structure.

We considered what this hypertext method means in the context of AI, both briefly in general, and in more detail specifically through three projects inspired by this line of thinking.

Reflecting over the past three decades of hypertext research, we can find a great variety of work presented. It can sometimes be challenging to draw the common "red thread" through this work and distill what it means for work to be "hypertextual" in nature. Our discussions on the goal of augmentation and the characteristics of first-class, context-dependent, and open ended structure provide one such framework for understanding our diverse community. Applying this method of inquiry to systems from other fields that solve problems outside of those normally considered part of the hypertext community provides a disciplined and structured way of continuing to draw our boundaries inclusively.

What are the consequences of considering hypertext as a method? What are the future research directions based on this approach? As summarized in the previous paragraph, our approach unveils a common hypertext research direction that unfolds in two different notions:

(1) The use of hypertext paradigms in different application domains, i.e., an "outbound perspective"

(2) The use of various other research or technologies for improving or extending hypertext systems, i.e., an "inbound perspective"

Future work targets both notions. Firstly, it includes rolling out hypertext paradigms to further application domains for which augmenting human intellect plays an essential role. In practice, this is any field which includes representing or communicating human thinking or creativity. In this paper we have already described this for the fields of software engineering (project HEIMDALL), entertainment (project DemoMedia), and industry context (project IWInxt). Further examples include journalism, education, collaborative work, note taking, among many others. This outbound perspective increases the number of applications of hypertext with its unique and special theme of human augmentation. As such it becomes distinguishable among other fields of research and defines its own niche.

Secondly, the inbound perspective takes advantage of current advances in various research directions for improving hypertext systems. It shows the complementarity of such fields to hypertext. This is an additional means to define the boundaries of hypertext and, thus, its related research communities. Similar to the outbound perspective, this helps us to define the niche for hypertext.

The discussion in this paper is directed toward to a common understanding of hypertext as a niche paradigm of high relevance and importance for a variety of fields of research and applications. This is in particular important in today's world where we encounter a huge increase of efforts in AI and, at the same time, a decreasing emphasis of human augmentation. The proposed point of view enables hypertext to become an application area for various technologies, including AI.

This strengthens hypertext and lets other fields benefit from it. As such, hypertext can neither be replaced or subsumed by its application domains nor by the technologies it uses. For example, as discussed in this paper, AI does not replace hypertext, but rather gets enhanced by being considered from a "hypertext perspective". This opens the use of hypertext for new fields of applications which otherwise would not be possible.

Considering hypertext as a method enables us to tie together a large number of communities that have their origin in hypertext. Furthermore, it lets us add more communities that relate directly or indirectly to the idea of augmentation or its various application. It encourages the hypertext community to further develop its paradigms and systems toward well known or newly discovered application areas or enhance them with new findings or technologies from other fields. This opens a sustainable view on hypertext in combination with computing technologies for the benefit of mankind, similar to what Douglas Engelbart envisioned: "more-rapid comprehension, better comprehension, the possibility of gaining a useful degree of comprehension in a situation that previously was too complex, speedier solutions, better solutions, and the possibility of finding solutions to problems that before seemed insoluble." [20]

\section{ACKNOWLEDGMENTS}

This work includes parts of the ODIN project, sub-project HEIM$D A L L$, funded by the German Federal Ministry of Education and Research (grant ID 03PSWKPD); the project Forschungsstelle Car Infotainment, sub-project DemoMedia, funded by the Bavarian State Ministry of Science and the Arts (grant ID 1547-RP-01); and the project IWInxt, funded by the Bavarian State Ministry of Science and the Arts (grant ID Kap. 1549 Tit. 547 78-2/2018).

\section{REFERENCES}

[1] Espen J. Aarseth. 1997. Cybertext: Perspectives on Ergodic Literature. Johns Hopkins University Press.

[2] Claus Atzenbeck, Daniel Roßner, and Manolis Tzagarakis. 2018. Mother - An Integrated Approach to Hypertext Domains. In Proceedings of the 29th ACM Conference on Hypertext and Social Media. ACM Press, 145-149. https://doi.org/ $10.1145 / 3209542.3209570$

[3] Claus Atzenbeck, Thomas Schedel, Manolis Tzagarakis, Daniel Roßner, and Lucas Mages. 2017. Revisiting Hypertext Infrastructure. In Proceedings of the 28th ACM Conference on Hypertext and Social Media 28th ACM Conference on Hypertext and Social Media. ACM Press, 35-44. https://doi.org/10.1145/3078714.3078718

[4] Belinda Barnet. 2018. Boosting Human Capability. In Proceedings of the 1st Workshop on Human Factors in Hypertext (HUMAN '18). ACM, 1. http://doi.acm. org/10.1145/3215611.3215615

[5] Belinda Barnet and Douglas C. Engelbart. 1999. First tape, Interview Between Doug Engelbart \& Belinda Barnet, 10th Nov, 1999. WWW. http: //www.fibreculture.org/reviews/barnet_engelbart.html 
[6] Tim Berners-Lee. 2000. Weaving the Web: The Original Design and Ultimate Destiny of the World Wide Web. Harper Business.

[7] Mark Bernstein. 1998. Patterns of hypertext. In Proceedings of the 9th ACM Conference on Hypertext and Hypermedia. ACM, 21-29. http://doi.acm.org/10. $1145 / 276627.276630$

[8] Mark Bernstein. 2009. On hypertext narrative. In Proceedings of the 20th ACM Conference on Hypertext and Hypermedia. ACM, 5-14. http://doi.acm.org/10. $1145 / 1557914.1557920$

[9] Mark Bernstein. 2010. Criticism. In Proceedings of the 21st ACM Conference on Hypertext and Hypermedia. ACM, 235-244. http://doi.acm.org/10.1145/1810617. 1810660

[10] Vannevar Bush. 1945. As we may think. The Atlantic Monthly 176, 1 (7 1945), 101-108. http://www.theatlantic.com/doc/194507/bush

[11] Licia Calvi. 2004. Adaptivity in Hyperfiction. In Proceedings of the Fifteenth ACM Conference on Hypertext and Hypermedia. ACM, New York, NY, USA, 163-170. http://doi.acm.org/10.1145/1012807.1012854

[12] CEN. 2001. Maintenance Terminology. European Standard EN 13306:2001. European Committee for Standardization.

[13] Jeff Conklin. 1987. Hypertext: an introduction and survey. Computer 20, 9 (1987), 17-41. http://dx.doi.org/10.1109/MC.1987.1663693

[14] Jeff Conklin and Michael L. Begeman. 1987. gIBIS: a hypertext tool for team design deliberation. In Proceedings of the ACM Conference on Hypertext. ACM Press, 247-251. http://doi.acm.org/10.1145/317426.317444

[15] Jeff Conklin, Albert Selvin, Simon Buckingham Shum, and Maarten Sierhuis. 2001. Facilitated hypertext for collective sensemaking: 15 years on from gIBIS In Proceedings of the 12th ACM Conference on Hypertext and Hypermedia. ACM Press, 123-124. http://doi.acm.org/10.1145/504216.504246

[16] Crossway Bibles (Ed.). 2001. Holy Bible. English Standard Version. Crossway.

[17] Robert-Alain de Beaugrande and Wolfgang Dressler. 1981. Introduction to Text Linguistics. Addison-Wesley.

[18] Steve DeRose, Eve Maler, David Orchard, and Norman Walsh. 2010. XML Linking Language (XLink) Version 1.1. Technical Report. W3C. http://www.w3.org/TR/ 2010/REC-xlink11-20100506/ W3C Recommendation 06 May 2010.

[19] J. Yellowlees Douglas. 1994. "How Do I Stop This Thing?": Closure and Indeterminacy in Interactive Narratives. In Hyper/Text/Theory, George P. Landow (Ed.) Johns Hopkins University Press.

[20] Douglas C. Engelbart. 1962. Augmenting Human Intellect: A Conceptual Framework. Summary Report AFOSR-3233. Standford Research Institute. http: //dougengelbart.org/content/view/138

[21] Douglas C. Engelbart. 1975. NLS Teleconferencing Features:: The Journal, and Shared-Screen Telephoning. In Proceedings of the CompCon 75 Conference. 173-176. http://dougengelbart.org/content/view/137/000/

[22] Gene Golovchinsky and Catherine C. Marshall. 2000. Hypertext Interaction Revisited. In Proceedings of the Eleventh ACM on Hypertext and Hypermedia. ACM, 171-179. http://doi.acm.org/10.1145/336296.336358

[23] Kaj Grønbæk and Randall H. Trigg. 1994. Design issues for a Dexter-based hypermedia system. Commun. ACM 37, 2 (2 1994), 40-49. http://doi.acm.org/10. $1145 / 175235.175238$

[24] Frank G. Halasz. 1987. Reflections on NoteCards: Seven issues for the next generation of hypermedia systems. In Proceedings of the ACM Conference on Hypertext. ACM, 345-365. http://doi.acm.org/10.1145/317426.317451

[25] Johndan Johnson-Eilola. 1993. Control and the Cyborg: Writing and Being Written in Hypertext. Fournal of Advanced Composition 13, 2 (1993), 381-399. http://www.jstor.org/stable/20865923

[26] Gregor Kiczales, John Lamping, Anurag Mendhekar, Chris Maeda, Cristina Lopes Jean-Marc Loingtier, and John Irwin. 1997. Aspect-oriented programming. In ECOOP'97 - Object-Oriented Programming, Mehmet Akşit and Satoshi Matsuoka (Eds.). Springer, 220-242.

[27] George P. Landow. 2006. Hypertext 3.0: Critical Theory and New Media in an Era of Globalization. Johns Hopkins.

[28] George P. Landow. 2009. Reconfiguring Writing. In Reading Hypertext, Diane Greco and Mark Bernstein (Eds.). Eastgate Systems, 89-98.

[29] Catherine C. Marshall and Frank M. Shipman. 1993. Searching for the missing link: discovering implicit structure in spatial hypertext. In Proceedings of the fifth ACM conference on Hypertext. ACM Press, 217-230. http://doi.acm.org/10.1145/ 168750.168826

[30] Jerome J. McGann. 1997. The Rationale of Hypertext. In Electronic Text: Investigations in Method and Theory, Kathryn Sutherland (Ed.). Oxford University Press, 19-46. https://dx.doi.org/10.1093/acprof:oso/9780198236634.001.0001

[31] Matthias Müller-Prove. 2002. Vision and Reality of Hypertext and Graphical User Interfaces. Technical Report FBI-HH-B-237/02. Universität Hamburg.

[32] Theodor Holm Nelson. 1965. Complex information processing: a file structure for the complex, the changing and the indeterminate. In Proceedings of the ACM 20th National Conference. ACM Press, 84-100. https://doi.org/10.1145/800197.806036

[33] Theodor Holm Nelson. 1987. Computer Lib/Dream Machines (2 ed.). Tempus Books.

[34] Theodor Holm Nelson. 1999. Xanalogical structure, needed now more than ever: parallel documents, deep links to content, deep versioning, and deep re-use.
Comput. Surveys 31, 4 (1999), article 33/pp. 1-32. http://doi.acm.org/10.1145/ 345966.346033

[35] Theodor Holm Nelson. 2015. What Box? In Intertwingled. The Work and Influence of Ted Nelson, Douglas R. Dechow and Daniele C. Struppa (Eds.). Springer, 133150. https://doi.org/10.1007/978-3-319-16925-5

[36] Jakob Nielsen. 1990. The art of navigating through hypertext. Commun. ACM 33, 3 (1990), 296-310. http://doi.acm.org/10.1145/77481.77483

[37] Peter J. Nürnberg. 2003. What is Hypertext?. In Proceedings of the Fourteenth ACM Conference on Hypertext and Hypermedia. ACM, 220-221. http://doi.acm. org $/ 10.1145 / 900051.900052$

[38] Peter J. Nürnberg, Kim C. Kristoffersen, David L. Hicks, and Uffe K. Wiil. 2005. EAD Revisited: First Experiences. In Proceedings of the 2005 Symposia on Metainformatics (MIS '05). ACM. http://doi.acm.org/10.1145/1234324.1234336

[39] Alexander C. Nwala, Michele C. Weigle, and Michael L. Nelson. 2018. Bootstrapping Web Archive Collections from Social Media. In Proceedings of the 29th on Hypertext and Social Media. ACM, 64-72. http://doi.acm.org/10.1145/3209542. 3209560

[40] H. Van Dyke Parunak. 1991. Don't link me in: Set based hypermedia for taxonomic reasoning. In Proceedings of the 3rd Annual ACM Conference on Hypertext. ACM Press, 233-242. http://doi.acm.org/10.1145/122974.122998

[41] Olav Reinert, Dirk Bucka-Lassen, Claus Aagaard Pedersen, and Peter J. Nürnberg. 1999. CAOS: a collaborative and open spatial structure service component with incremental spatial parsing. In Proceedings of the 10th ACM Conference on Hypertext and Hypermedia. ACM Press, 49-50. http://doi.acm.org/10.1145/294469. 294484

[42] Daniel Roßner and Claus Atzenbeck. 2018. Spatial Hypertext for End-User Development Tools. In Proceedings of the 1st Workshop on Human Factors in Hypertext (HUMAN'18). ACM, 9-15. http://doi.acm.org/10.1145/3215611.3215612

[43] Daniel Roßner, Claus Atzenbeck, and Tom Gross. 2019. Visualization of the relevance: using physics simulations for encoding context. In Proceedings of the 30th ACM Conference on Hypertext and Social Media (HT '19). ACM. In print.

[44] Hohyon Ryu, Matthew Lease, and Nicholas Woodward. 2012. Finding and Exploring Memes in Social Media. In Proceedings of the 23rd ACM Conference on Hypertext and Social Media. ACM, 295-304. http://doi.acm.org/10.1145/2309996.2310044

[45] Amin Salehi, Mert Ozer, and Hassan Davulcu. 2018. Sentiment-driven Community Profiling and Detection on Social Media. In Proceedings of the 29th on Hypertext and Social Media. ACM, 229-237. http://doi.acm.org/10.1145/3209542.3209565

[46] Thomas Schedel and Claus Atzenbeck. 2016. Spatio-Temporal Parsing in Spatial Hypermedia. In Proceedings of the 27th ACM Conference on Hypertext and Social Media. ACM, 149-157. http://dx.doi.org/10.1145/2914586.2914596

[47] Manolis Tzagarakis, Dimitris Avramidis, Maria Kyriakopoulou, Monica M. C. Schraefel, Michalis Vaitis, and Dimitris Christodoulakis. 2003. Structuring primitives in the Callimachus component-based open hypermedia system. Fournal of Network and Computer Applications 26, 1 (2003), 139-162. http://dx.doi.org/10. 1016/S1084-8045(02)00064-4

[48] Manolis Tzagarakis, Michail Vaitis, and Nikos Karousos. 2006. Designing domainspecific behaviours in structural computing. New Review of Hypermedia and Multimedia 2 (12 2006), 113-142. http://dx.doi.org/10.1080/13614560601053527

[49] Andries van Dam. 1988. Hypertext '87: Keynote Address. Communication of the ACM 31, 7 (July 1988), 887-895. http://doi.acm.org/10.1145/48511.48519

[50] William Van Lepthien and Kenneth M. Anderson. 2004. Unifying structure, behavior, and data with themis types and templates. In Proceedings of the 15th ACM Conference on Hypertext and Hypermedia. ACM, 256-265. http://doi.acm. org/10.1145/1012807.1012870

[51] Jill Walker. 2002. Links and Power: The Political Economy of Linking on the Web. In Proceedings of the Thirteenth ACM Conference on Hypertext and Hypermedia. ACM, 72-73. http://doi.acm.org/10.1145/513338.513358

[52] Jill Walker. 2005. Feral hypertext: when hypertext literature escapes control. In HYPERTEXT '05: Proceedings of the sixteenth ACM conference on Hypertext and hypermedia. ACM, 46-53. http://doi.acm.org/10.1145/1083356.1083366

[53] Noah Wardrip-Fruin. 2004. What Hypertext is. In Proceedings of the Fifteenth ACM Conference on Hypertext and Hypermedia. ACM, 126-127. http://doi.acm. org/10.1145/1012807.1012844

[54] Zhongyu Wei, Yulan He, Wei Gao, Binyang Li, Lanjun Zhou, and Kam-fai Wong. 2013. Mainstream Media Behavior Analysis on Twitter: A Case Study on UK General Election. In Proceedings of the 24th ACM Conference on Hypertext and Social Media. ACM, 174-178. http://doi.acm.org/10.1145/2481492.2481512

[55] E. James Whitehead. 2001. Design spaces for link and structure versioning. In Proceedings of the 12th ACM Conference on Hypertext and Hypermedia. ACM Press, 195-204. http://doi.acm.org/10.1145/504216.504265

[56] Uffe Kock Wiil. 2005. Hypermedia technology for knowledge workers: a vision of the future. In Proceedings of the 16th ACM Conference on Hypertext and Hypermedia. ACM, 4-6. http://doi.acm.org/10.1145/1083356.1083358

[57] Uffe K. Wiil, David L. Hicks, and Peter J. Nürnberg. 2001. Multiple open services: a New Approach to Service Provision in Open Hypermedia Systems. In Proceedings of the 12th ACM Conference on Hypertext and Hypermedia. ACM Press, 83-92. http://doi.acm.org/10.1145/504216.504241 